\title{
TENDÊNCIAS HIDROLÓGICAS NA REGIÃO DO ALTO RIO MOGI-GUAÇU, SUL DE MINAS GERAIS
}

Dr. Mateus Ricardo Nogueira Vilanova ${ }^{1}$

\section{Resumo}

Variações temporais do comportamento hidrológico de uma região podem ser decorrentes de alterações climáticas ou do uso e ocupação do solo em bacias hidrográficas, sendo o seu entendimento fundamental para o gerenciamento e planejamento de recursos hídricos. O presente trabalho avalia a existência de tendências monotônicas em séries de vazão e chuva na região do Alto Rio Mogi-Guaçu, localizada no sul do Estado de Minas Gerais. Para isso, foi aplicado o teste não-paramétrico de MannKendall a séries representativas de vazão média anual de duas estações fluviométricas, e o teste de MannKendall Regional a séries de chuva total anual de duas estações pluviométricas, ambos no período de 1973 a 2006. Os resultados demonstraram a existência de tendências estatisticamente significativas de redução da chuva e da vazão na região do Alto Rio Mogi-Guaçu durante o período analisado.

Palavras-chave: Alterações hidrológicas. Chuva. Vazão fluvial.

\section{Introdução}

As evidências de mudanças climáticas globais aumentaram a relevância de estudos sobre tendências hidrológicas. Segundo o World Water Assessment Programme (2009), os maiores impactos dessas mudanças sobre a humanidade e ecossistemas ocorrem através da água, seja na forma de secas e inundações severas, alterações da disponibilidade quantitativa e qualitativa para usos múltiplos ou do aumento da ocorrência de doenças de veiculação hídrica. Além dessa abordagem atual, variações no comportamento hidrológico podem ocorrer, também, devido às mudanças do uso e ocupação do solo em bacias hidrográficas, alterando a resposta hidrológica de diversos processos, como, por exemplo, escoamento superficial, infiltração e evapotranspiração.

O estudo de alterações hidrológicas em bacias hidrográficas é fundamental para o planejamento de recursos hídricos, pois estas alterações influenciam diretamente a disponibilidade hídrica (quantitativa e qualitativa) para os usos múltiplos, como abastecimento público, agricultura e geração de energia.

O Rio Mogi-Guaçu (RMG) é um dos principais cursos d'água da bacia do Rio Grande, cuja nascente se localiza no município de Bom Repouso, sul de Minas Gerais. O trecho mineiro da bacia do RMG integra a Unidade de Planejamento e Gestão de Recursos Hídricos dos Afluentes dos Rios Mogi-Guaçu e Pardo (GD6) do Instituto Mineiro de Gestão das Águas (IGAM), após o qual o rio adentra em território paulista, dentro da Unidade de Gerenciamento de Recursos Hídricos (UGRHI 09) do Estado de São Paulo.

Na GD6, na qual foi desenvolvido o presente estudo, os principais usos da água são referentes ao abastecimento público, diluição de efluentes, irrigação e pecuária, geração de energia (IGAM, 2010), enquanto na UGRHI09 predominam a irrigação (47\% do total) e o uso industrial (36\%) (CBH MOGI, 2013).

A importância econômica nacional das GD6 e UGRHI, associada à grande dependência de recursos hídricos das atividades regionais, como por exemplo as extensas culturas de cana-de-açúcar e laranja do interior paulista, motivaram o presente estudo. 0 objetivo do trabalho é detectar tendências monotônicas, de aumento ou diminuição, em séries de chuva total anual e vazão média anual na região do Alto RMG, no trecho mineiro de sua bacia hidrográfica. A redução ou aumento dos padrões de chuva e vazão nessa região podem resultar em consequências diretas para os trechos de jusante da bacia, na qual se concentram as principais demandas de água, como por exemplo, a redução da disponibilidade hídrica para irrigação e abastecimento público.

${ }^{1}$ UNESP - Universidade Estadual Paulista, Faculdade de Engenharia de Guaratinguetá (FEG), Programa de Pós-graduação em Engenharia Mecânica (Transmissão e Conversão de Energia) e Departamento de Engenharia Civil, 333, Dr. Ariberto Pereira da Cunha Av. Guaratinguetá, SP, Brasil. Tel:: 5535 


\section{Material e métodos}

\subsection{Estações e séries hidrológicas utilizadas}

Séries de chuva total diária e vazão média diária foram utilizadas para calcular as chuvas totais mensais e vazões médias mensais e, posteriormente, os respectivos valores anuais. Tais séries foram obtidas no servidor Hidroweb ${ }^{2}$ da Agência Nacional de Águas (ANA), tendo sido considerados somente meses com registros diários completos. A seleção das estações ocorreu em função da localização geográfica (dentro da área de estudo, a bacia hidrográfica do RMG, na unidade GD6, no caso de estações pluviométricas, e instaladas no RMG, no caso de estações fluviométricas), extensão (mínimo de 30 anos contínuos), atualidade, e integridade (razão entre o número de meses com registros diários completos e o número total de meses no período de análise) das séries de dados. A Tabela 1 apresenta as principais características das estações selecionadas, que atenderam aos critérios descritos, e a Figura 1 o mapa de localização destas estações.

Tabela 1. Estações hidrológicas utilizadas no estudo.

\begin{tabular}{|c|c|c|c|c|c|c|}
\hline & \multirow{2}{*}{ Estação } & \multicolumn{2}{|c|}{$\begin{array}{c}\text { Coordenadas } \\
\text { WGS84 }\end{array}$} & \multirow{2}{*}{$\begin{array}{c}\text { Área de } \\
\text { drenagem } \\
{\left[\mathrm{km}^{2}\right]}\end{array}$} & \multirow{2}{*}{ Município } & \multirow[t]{2}{*}{ Integridade } \\
\hline & & Lat. & Lon. & & & \\
\hline \multirow{2}{*}{ Chuva } & 2246052 & $-22^{\circ} 17^{\prime} 19^{\prime \prime}$ & $-46^{\circ} 37^{\prime} 09^{\prime}$ & - & Jacutinga & $98,2 \%$ \\
\hline & 2246056 & $-22^{\circ} 19^{\prime} 16^{\prime \prime}$ & $-46^{\circ} 19^{\prime} 46^{\prime \prime}$ & - & Inconfidentes & $94,9 \%$ \\
\hline \multirow{2}{*}{ V azão } & 61861000 & $-22^{\circ} 19^{\prime} 06^{\prime \prime}$ & $-46^{\circ} 19^{\prime} 19^{\prime \prime}$ & 463 & Inconfidentes & $97,7 \%$ \\
\hline & 61865000 & $-22^{\circ} 16^{\prime} 12^{\prime \prime}$ & $-46^{\circ} 36^{\prime} 15^{\prime \prime}$ & 918 & Jacutinga & $94,4 \%$ \\
\hline
\end{tabular}

Fonte: Elaboração do autor

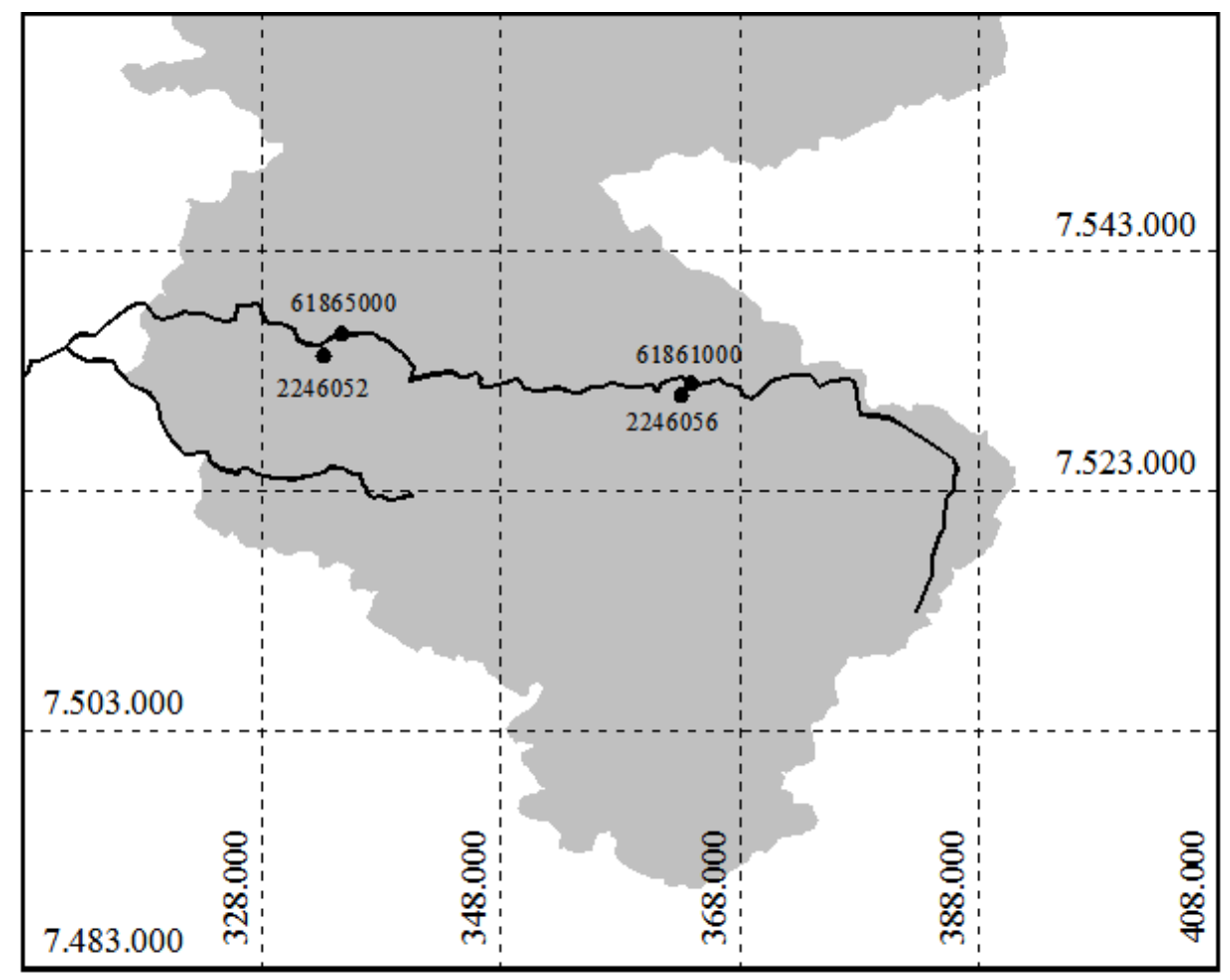

Figura 1. Trecho da GD6 estudado e localização das estações hidrológicas (coordenadas UTM, zona 23K). Fonte: Elaboração do autor 
Falhas nas séries de chuva foram preenchidas através de regressão linear entre os totais mensais das duas estações consideradas (TUCCI, 2004), e apresentaram ajuste satisfatório $\left(R^{2}=0,72\right)$. As séries de vazão foram, inicialmente, preenchidas através de ajuste de funções polinomiais quadráticas entre vazões médias mensais das duas séries consideradas, que também apresentaram bom ajuste $\left(R^{2}>0,87\right)$. Por conta das falhas mútuas nas duas estações nos anos hidrológicos de 1991, 1992 e 1993, replicou-se a inicial abordagem, considerando, porém, uma estação auxiliar (61879000), instalada no Rio do Peixe, um dos principais afluentes do RMG. Além da proximidade entre as estações 61861000 e 61865000 e a estação 61879000 (aproximadamente $40 \mathrm{~km}$ e $29 \mathrm{~km}$, respectivamente), a bacia do Rio do Peixe apresenta grande semelhança física e climática em relação ao trecho da bacia do RMG estudado, semelhança essa representada pelo bom ajuste das funções de transposição entre essas estações $\left(R^{2}=\right.$ 0,83 e $R^{2}=0,91$ para as estações 61861000 e 61865000 , respectivamente). Em ambos os casos, as falhas nas séries localizam-se nos intervalos de dados utilizados no ajuste das funções de transposição, não tendo ocorrido extrapolação.

O ano hidrológico típico do sudeste brasileiro foi considerado no cálculo dos valores de chuva total anual e vazão média anual, iniciando em outubro (início do período chuvoso) e terminando em setembro do ano seguinte. Dada a disponibilidade de dados, foi considerado o período de análise de 1973 a 2006 (33 anos) para todas as séries (fluviométricas e pluviométricas). A Tabela 2 apresenta as estatísticas descritivas das séries utilizadas: média, desvio-padrão (DP), coeficiente de variação (CV), mínimo (Mín.) e máximo (Máx).

Tabela 2. Estatísticas descritivas das séries hidrológicas utilizadas.

\begin{tabular}{ccccccc}
\hline Estação & $\begin{array}{c}\text { Chuva média } \\
\text { anual }[\mathrm{mm}]\end{array}$ & $\begin{array}{c}\text { DP } \\
{[\mathrm{mm}]}\end{array}$ & CV & $\begin{array}{c}\text { Mín. } \\
{[\mathrm{mm}]}\end{array}$ & $\begin{array}{c}\text { Máx. } \\
{[\mathrm{mm}]}\end{array}$ \\
\hline \multirow{2}{*}{ Chuva } & 2246052 & 1727 & 307 & $18 \%$ & 1224 & 2596 \\
& 2246056 & 1492 & 312 & $21 \%$ & 998 & 2378 \\
\hline \multirow{6}{*}{ Vazão } & Estação & $\begin{array}{c}\text { Vazão média de } \\
\text { longo termo } \\
{\left[\mathrm{m}^{3} \cdot \mathbf{s}^{-1}\right]}\end{array}$ & $\begin{array}{c}\text { DP } \\
{\left[\mathrm{m}^{3} \cdot \mathrm{s}^{-1}\right]}\end{array}$ & $\mathbf{C V}$ & $\begin{array}{c}\text { Mín. } \\
{\left[\mathrm{m}^{3} . \mathrm{s}^{-1}\right]}\end{array}$ & $\begin{array}{c}\text { Máx. } \\
{\left[\mathrm{m}^{3} \cdot \mathrm{s}^{-1}\right]}\end{array}$ \\
& 6186100 & 10,6 & 2,8 & $27 \%$ & 5,4 & 22,2 \\
& 6186500 & 19,2 & 4,6 & $24 \%$ & 11,8 & 36,8 \\
\hline
\end{tabular}

Fonte: Elaboração dos autores

\subsection{Teste de Mann-Kendall}

A detecção de tendências foi realizada através dos métodos de Mann-Kendall e Mann-Kendall Regional, amplamente utilizados na análise de variáveis hidrológicas (por exemplo, BAO et al. 2012; ZHANG et al. 2011; XU et al. 2010; WILSON et al. 2010; BRABETS; WALVOORD, 2009; MARENGO; ALVES, 2005; BURN et al. 2004; LINDSTROM; BERGSTROM, 2004).

As bases teóricas do teste de Mann-Kendall foram propostas por Mann (1945) e Kendall (1975). 0 teste detecta tendências monotônicas em séries temporais, sendo um método não-paramétrico (HIPEL; MCLEOD, 1994; XU et al. 2010) que não requer que seja assumida a normalidade da série (HELSEL; FRANS, 2006). A Equação 1 apresenta a estatística de teste de Mann-Kendall, que considera a hipótese nula $\left(H_{0}\right)$ de que os dados vem de uma população na qual as variáveis aleatórias são independentes e identicamente distribuídas, enquanto a hipótese alternativa $\left(\mathrm{H}_{1}\right)$ representa a existência de tendência monotônica. Nas equações 1 e 2, S é a estatística de teste de Mann-Kendall, n o tamanho da amostra, sgn o sinal da diferença de valores (de chuva ou vazões) subsequentes. Nas equações 3 e 4, E[S] é a media de $S$, Var[S] é a variância de S, p é o número de grupos amarrados (conjunto de dados da amostra com os mesmos valores) e t é o número de dados do grupo amarrado de ordem j. Z (Equação 5) é a estatística de teste para amostrar com $n>10$, que se aproxima da distribuição normal.

$$
\begin{aligned}
& S=\sum_{k=1}^{n-1} \sum_{j=k+1}^{n} \operatorname{sgn}\left(x_{j}-x_{k}\right) \\
& \operatorname{sgn}(x)=+1, x>0 \\
& \operatorname{sgn}(x)=0, x=0 \\
& \operatorname{sgn}(x)=-1, x<0
\end{aligned}
$$




$$
\mathrm{E}[\mathrm{S}]=0
$$

$$
\begin{aligned}
& \operatorname{Var}[\mathrm{S}]=\frac{\left(\left\{\mathrm{n}(\mathrm{n}-1)(2 \mathrm{n}+5)-\sum_{\mathrm{j}=1}^{\mathrm{p}} \mathrm{t}_{\mathrm{j}}\left(\mathrm{t}_{\mathrm{j}}-1\right)\left(2 \mathrm{t}_{\mathrm{j}}\right\}+5\right)\right.}{18} \\
& \mathrm{Z}=\frac{\mathrm{S}-1}{[\operatorname{Var}(\mathrm{S})]_{2}}, \text { para } \mathrm{S}>0 \\
& \mathrm{Z}=0, \operatorname{para} \mathrm{S}=0 \\
& \mathrm{Z}=\frac{\mathrm{S}+1}{[\operatorname{Var}(\mathrm{S})]_{2}}, \operatorname{para} \mathrm{S}<0
\end{aligned}
$$

No teste de Mann-Kendall Regional (HELSEL; FRANS, 2006), a estatística de teste regional (S) é dada pela soma das estatísticas individuais, de acordo com a Equação 6, na qual m é o número de locais da região e $\mathrm{S}_{\llcorner}$é a estatística de Mann-Kendall para o local L. Assim como no teste individual (local), a estatística pode ser convertida para $Z_{r}$ (Equação 7), quando a amostra $n>10$, que se aproxima da distribuição normal. A média de $S_{\mathrm{r}}$ é calculada através da Equação 8, e seu desvio padrão pela Equação 9 , sendo $\mathrm{n}_{\llcorner}$o número de dados do local de ordem $L$ para os $m$ locais da região.

$$
\begin{aligned}
& \mathrm{S}_{\mathrm{r}}=\sum_{\mathrm{L}=1}^{\mathrm{m}} \mathrm{S}_{\mathrm{L}} \\
& \mathrm{Z}_{\mathrm{r}}=\frac{\mathrm{S}_{\mathrm{r}}-1}{\sigma_{\mathrm{S}_{\mathrm{r}}}}, \text { para } \mathrm{S}_{\mathrm{r}}>0 \\
& \mathrm{Z}_{\mathrm{r}}=0, \text { ara } \mathrm{S}_{\mathrm{r}}=0 \\
& \mathrm{Z}_{\mathrm{r}}=\frac{\mathrm{S}_{\mathrm{r}}+1}{\sigma_{\mathrm{S}_{\mathrm{r}}}}, \text { para } \mathrm{S}_{\mathrm{r}}<0 \\
& \mu\left(\mathrm{S}_{\mathrm{r}}\right)=0 \\
& \sigma_{\mathrm{S}_{\mathrm{r}}}=\sqrt{\sum_{\mathrm{L}=1}^{\mathrm{m}}\left(\frac{\mathrm{n}_{\mathrm{L}}}{18}\right)\left(\mathrm{n}_{\mathrm{L}}-1\right)\left(2 \mathrm{n}_{\mathrm{L}}+5\right)}
\end{aligned}
$$

Uma região é definida como uma área especial contínua com características físicas semelhantes, ou mesmo por várias áreas discretas com as mesmas características físicas (HELSEL; FRANS, 2006).

0 teste de Mann-Kendall assume que os dados utilizados são independentes, não existindo correlação serial de curto prazo entre eles (HELSEL et al., 2006). Caso haja autocorrelação serial na série, deve-se aplicar o procedimento de pré-branqueamento para sua remoção (BAYAZIT; ONOZ, 2007). A detecção de autocorrelação serial é realizada através do cálculo da função de autocorrelação para o lag 1 $\left(r_{1}\right)$, considerando o nível de significância de $5 \%$ (BURN et al., 2004; WILSON et al., 2010).

Aplicou-se o teste de Mann-Kendall às séries de vazão, e sua versão regional, teste de MannKendall Regional, às séries de chuva. Através destes métodos, foram calculadas as estatísticas $\mathrm{S}$ e Z, o coeficiente de correlação de Kendall $(\tau)$, o valor-p dos testes e a função linear representativa das tendências. Considerou-se na avaliação dos resultados o nível de significância de $5 \%$. A declividade da função de tendência (que representa a magnitude da variação da variável considerada) corresponde ao 
estimador de declividade de Theil-Sen ( $\beta$ ) (THEIL, 1950; SEN, 1968), calculado através da Equação 10, na qual x é o valor da variável (chuva ou vazão), e i e j são as ordens referentes ao tempo na série temporal.

$$
\beta=\operatorname{mediana}\left(\frac{\mathrm{x}_{\mathrm{j}}-\mathrm{x}_{\mathrm{i}}}{\mathrm{j}-\mathrm{i}}\right) \quad \text { para todo } \mathrm{i}<\mathrm{j}<\mathrm{n}
$$

\section{Resultados e discussão}

Os resultados da Tabela 3 demonstram que não há autocorrelação nas séries estudadas ( $r_{1}$ dentro dos intervalos de confiança), o que permitiu a aplicação dos testes de Mann-Kendall às séries originais.

Tabela 3. Análise de autocorrelação nas séries hidrológicas utilizadas.

\begin{tabular}{ccc|cc} 
& \multicolumn{2}{c}{ Chuva } & \multicolumn{2}{c}{ Vazão } \\
Estação & 2246052 & $\mathbf{2 2 4 6 0 5 6}$ & $\mathbf{6 1 8 6 1 0 0 0}$ & $\mathbf{6 1 8 6 5 0 0 0}$ \\
$\mathbf{r}_{1}$ & 0,2068 & 0,1686 & 0,2482 & 0,3037 \\
\pm IC 95\% & 0,3482 & 0,3482 & 0,3482 & 0,3482 \\
\hline
\end{tabular}

Fonte: Elaboração dos autores

Verifica-se nas tabelas 4 e 5 que os valores-p dos três testes executados são inferiores ao nível de significância considerado referência $(\alpha=0,05)$, o que leva à rejeição de $H_{0}$. Com isso, conclui-se que existem tendências estatisticamente significativas nas vazões do RMG e na chuva das estações de sua área avaliada. Estas tendências são negativas, resultando numa redução da vazão $(\beta)$ de $0,089 \mathrm{~m}^{3} \cdot \mathrm{s}^{-1}$.ano ${ }^{-1}$ na estação 61861000 e $0,1392 \mathrm{~m}^{3} \cdot \mathrm{s}^{-1}$.ano ${ }^{-1}$ na estação 61865000 . Estes valores representam reduções anuais de $0,84 \%$ e $0,73 \%$ em relação às vazões médias das estações 61861000 e 61865000 , respectivamente. A redução média $(\Delta)$ da chuva nas estações avaliadas é de 9,8 mm.ano ${ }^{-1}$. Devido à inexistência de dados entre 2006 e 2013 das estações consideradas no estudo, não é possível determinar a persistência ou alteração das tendências até este último ano.

Tabela 4. Resultados do teste de Mann-Kendall para vazões do RMG.

\begin{tabular}{cccccc}
\hline Estação & $\tau$ & $\mathbf{S}$ & $\mathbf{Z}$ & Valor-p & $\beta$ \\
61861000 & $-0,275$ & -145 & $-2,233$ & 0,0255 & $-0,0890$ \\
61865000 & $-0,256$ & -135 & $-2,077$ & 0,0378 & $-0,1392$ \\
\hline
\end{tabular}

Fonte: Elaboração dos autores

Tabela 5. Resultados do teste de Mann-Kendall Regional para chuva na região do RMG.

\begin{tabular}{cccccc}
\hline Estações & $\tau$ & $\mathbf{S}$ & $\mathbf{Z}$ & Valor-p & $\Delta$ \\
2246052 & $-0,226$ & -239 & $-2,608$ & 0,0091 & $-9,8$ \\
2246056 & & & & & \\
\hline
\end{tabular}

Fonte: Elaboração dos autores

A existência de tendências de mesma natureza (negativa) tanto na chuva quanto na vazão da região sugere que as tendências identificadas possuem uma componente de origem climática — afetando inicialmente a chuva da região e possivelmente gerando uma resposta de mesma natureza nas vazões. Esta componente pode ser resultado de processos climáticos globais ou de mesoescala, refletindo alterações climáticas regionais.

Alterações físicas, mais especificamente do uso e ocupação do solo na bacia hidrográfica estudada, podem compor uma segunda componente associada às tendências negativas detectadas. Tais 
alterações têm influência direta sobre os processos de conversão precipitação-vazão da bacia, alterando as taxas dos variados processos hidrológicos (por exemplo, infiltração e interceptação) influentes sobre a resposta hidrográfica nas seções fluviais/estações fluviométricas analisadas.

Além da influência direta sobre o escoamento superficial da bacia, diferentes tipos de cobertura do solo podem causar alterações, também na precipitação da região. Segundo Nuñez et al. (2008), forçantes locais de superfície sobre as mudanças climáticas são de difícil detecção, mas estudos recentes indicam que o impacto das mudanças generalizadas do uso do solo não devem ser ignorados. Vilanova (2014), por exemplo, analisou tendências hidrológicas na Bacia do Rio Paraibuna (SP), tendo encontrado tendências distintas em duas estações pluviométricas distantes entre si apenas $35 \mathrm{~km}$. Segundo o autor, a principal hipótese para o comportamento distinto da chuva das duas estações avaliadas é a influência direta do uso e ocupação do solo, sendo que uma das estações localiza-se em um trecho de forte expansão agrícola, e a outra, na qual não foram detectadas tendências, localiza-se dentro de uma área de preservação ambiental florestal.

Vilanova (2014) apresentou, ainda, diversas referências da literatura que demonstraram a influência do uso e ocupação do solo sobre a chuva regional, em escalas de até $10 \mathrm{~km}$. Um trabalho recente que aborda a questão foi desenvolvido por Murgida et al. (2014), que abordaram as inter-relações entre tendências de precipitação, mudança no uso do solo e adaptações na região do Chaco (Argentina). Segundo os autores, as mudanças identificadas na chuva coincidiram com um período de degradação florestal na região do Chaco devido às atividades extrativistas.

As características e a delimitação do presente estudo não permitem determinar se a redução da chuva e vazão verificada na região do Alto RMG é decorrente de componentes climáticas, de uso e ocupação do solo ou de uma associação de ambas, e ainda, qual é a participação de cada componente sobre as taxas de variação calculadas. Vislumbram-se, desta forma, possibilidades de novos estudos avaliando, especificamente, as causas das tendências hidrológicas detectadas, bem como suas implicações em termos de gerenciamento de recursos hídricos na região.

Em termos de vazão, uma terceira componente poderia explicar a redução de seus valores ao longo do tempo: o aumento da captação para usos múltiplos. Moraes et al. (1998), por exemplo, detectaram tendências significativas na vazão, precipitação e evapotranspiração na bacia do Rio Piracicaba, tendo associado as reduções da vazão principalmente à exportação de água para a Região Metropolitana de São Paulo através do Sistema Cantareira.

\section{Conclusão}

A chuva e as vazões na região do Alto Rio Mogi-Guaçu apresentaram tendências estatisticamente significativas de redução no período analisado.

\section{Agradecimentos}

À Coordenação de Aperfeiçoamento de Pessoal de Nível Superior (CAPES), pela bolsa de pós-doutorado do Programa Nacional de Pós-doutorado (PNPD).

\section{Hydrological trends in the upper Mogi-Guaçu River Region, South of Minas Gerais State}

\section{Abstract}

Temporal variations of the hydrological behavior of a region may be due to changes of the climate or of land use and occupation in watersheds, and its understanding is essential for water resources planning and management. This paper assesses the existence of monotonic trends in streamflow and rainfall series in the Upper Mogi-Guaçu River region, located in the south of Minas Gerais State. For this, the 
nonparametric Mann-Kendall test was applied to representative series of mean annual streamflow (of two streamflow stations), and the Regional Mann-Kendall test was applied to series of total annual rainfall (of two rainfall stations), both of period between 1973 and 2006. The results demonstrated the existence of statistically significant trends of reduction of the rainfall and streamflow in the study region.

Keywords: Hydrological Change. Rainfall. Streamflow.

\section{Referências bibliográficas}

BAO, Z.; et al. Attribution for decreasing streamflow of the Haihe River basin, northern China: Climate variability or human activities? Journal of Hydrology, Amsterdam, v. 460-461, p. 117-129, 2012.

BAYAZIT, M.; ÖNÖZ, B. To prewhiten or not to prewhiten in trend analysis? Hydrological Sciences Journal, Londres, v. 52, n. 4, p. 611-624, 2007.

BRABETS, T. P.; WALVOORD, M. A. Trends in streamflow in the Yukon River Basin from 1944 to 2005 and the influence of the Pacific Decadal Oscillation. Journal of Hydrology, Amsterdam, v. 371, p. 108-119, 2009.

BURN, D. H.; CUNDERLIK, J. M.; PIETRONIRO, A. Hydrological trends and variability in the Liard River basin. Hydrological Sciences Journal, Londres, v. 49, n. 1, p. 53-67, 2004.

COMITÊ DA BACIA HIDROGRÁFICA DO RIO MOGI GUAÇU. Relatório de situação dos recursos hídricos 2012 (ano base 2011): UGRHI - 09 CBH-Mogi. São João da Boa Vista: CBH MOGI, 2013.

HELSEL, D. R.; FRANS, L. M. Regional Kendall test for trend. Environmental Science and Technology, S.I., v. 40, n. 13, p. 4066-4070, 2006.

HELSEL, D. R.; MUELLER, D. K.; SLACK, J. R. Computer program for the Kendall family of trend tests: U. S. Geological Survey Scientific Investigations Report 2005-5275. Reston: USGS; 2006.

HIPEL, K. W.; MCLEOD, A. I. Time series modelling of water resources and environmental systems. Amsterdam: Elsevier Science, 1994.

INSTITUTO MINEIRO DE GESTÃO DAS ÁGUAS. Plano diretor de recursos hídricos dos afluentes mineiros dos Rios Mogi-Guaçu/Pardo: Relatório síntese. Belo Horizonte: IGAM, 2010.

KENDALL, M. G. Rank correlation methods. 4. ed. Londres: Charles Griffin, 1975.

LINDSTRÖM, G.; BERGSTRÖM, S. Runoff trends in Sweden 1807-2002. Hydrological Sciences Journal, Londres, v. 49, n. 1, p. 69-83, 2004.

MARENGO, J. A.; ALVES, L. M. Tendências hidrológicas da Bacia do Rio Paraíba do Sul. Revista Brasileira de Meteorologia, São Paulo, v. 20, n. 2, p. 215-226, 2005.

MANN, H. B. Non-parametric test against trend. Econometrica, S. I., v. 13, n. 3, p. 245-259, 1945.

MORAES, J. M., et al. Trends in hydrological parameters of a SouthernBrazilian watershed and its relation to human induced changes. Water Resources Management, Dordrecht, v. 12, n. 4, p. 295-311, 1998.

MURGIDA, A. M.; GONZALEZ, M. H.; TIESSEN, H. Rainfall trends, land use change and adaptation in the Chaco salteño region of Argentina. Regional Environmental Change (on-line), 2014. DOI:10.1007/s10113-013-0581-9 
NUÑEZ, M. N., et al. Impact of land use and precipitation changes on surface temperature trends in Argentina. Journal of Geophysical Research: Atmospheres, Malden, v. 113, n. D6, p. 1-11, 2008.

SEN, P. K. Estimates of the regression coefficients based on Kendall's tau. Journal of American Statistical Association, Alexandria, v. 63, p. 1379-1389, 1968.

THEIL, H. A rank-invariant method of linear and polynomial analysis, part 3. Nederlandse Akademie van Wettenschappen Proceedings, S. I., v. 53, p. 1397-1412, 1950.

TUCCI, C. E. M. Hidrologia: ciência e aplicação. 3. ed., Porto Alegre: UFRGS, 2004.

VILANOVA, M. R. N. Tendências hidrológicas anuais e sazonais na Bacia do Rio Paraibuna, Parque Estadual da Serra do Mar (SP). Sociedade \& Natureza (UFU. On-line), Uberlândia, v. 26, n. 2, p. 301316, 2014.

$\mathrm{XU}, \mathrm{Z}$; ; et al. Trends of major hydroclimatic variables in the Tarim River basin during the past 50 years. Journal of Arid Environments, Amsterdam, v. 74, p. 256-267, 2010.

WILSON, D.; HISDAL, H.; LAWRANCE, D. Has streamflow changed in the Nordic countries? Recent trends and comparisons to hydrological projections. Journal of Hydrology, Amsterdam, v. 394, p. 334-346, 2010.

WORLD WATER ASSESSMENT PROGRAMME. The United Nations World Water Development Report 3: Water in a Changing World. Paris: UNESCO; London: Earthscan, 2009.

ZHANG, Y.; et al. Analysis of impacts of climate variability and human activity on streamflow for a river basin in northeast China. Journal of Hydrology, Amsterdam, v. 410, p. 239-247, 2011.

\section{Histórico editorial}

Recebido em: 27/07/13

Aceito em: 23/04/14 